Liebe SÄZ-Leserinnen und -Leser, Missverständnisse sind vorprogrammiert, wenn zwei Menschen versuchen, einander zu verstehen. Selbst banale Aussagen werden vor den verschiedenen Erfahrungshorizonten der Beteiligten zu einer reichen Quelle von Irrtümern. So scheint es uns wertvoll, rund um Managed Care die Begriffe zu klären, damit man zumindest die gleiche Sprache spricht. Selbst das Wort «Managed Care» ist verschwun- den: Man spricht heute von «integrierter Versorgung». Das Thema wird in den nächsten Monaten politisch heiss: die Junisession des Parlaments befasst sich nämlich mit der KVG-Reform «Managed Care». Wir beginnen heute mit der «Budgetmitverantwortung». Gute Lektüre!

Dr. med. Ignazio Cassis, Vorstandsmitglied Forum Managed Care, Vizepräsident der FMH und Nationalrat

\title{
Budgetmitverantwortung in der integrierten Versorgung
}

\section{Peter Berchtold}

PD Dr. med.,

Präsident Forum Managed Care
Korrespondenz:

Forum Managed Care Pflanzschulstrasse 3

CH-8400 Winterthur Tel. 0522350553 Fax 0522351920

info@fmc.ch
Rund 100 Ärztenetze und HMO (Health Maintenance Organization) gibt es heute in der Schweiz, und knapp die Hälfte aller Grundversorgerinnen und Grundversorger engagieren sich in diesen Ärztenetzen. Etwa 80\% der Ärztenetze haben in ihren Verträgen mit den Krankenversicherern eine Form der Budgetmitverantwortung (BMV) vereinbart. Die BMV ist in der 20-jährigen Entwicklungsgeschichte der Integrierten Versorgung zu einem bedeutenden Element der Schweizer Ärztenetze geworden. BMV meint die institutionelle Verantwortung des Ärztenetzes (und nicht etwa des einzelnen Arztes!), für eine bestimmte Gruppe von Patienten ein vereinbartes Kostenziel einzuhalten; von BudgetMITverantwortung spricht man, weil das Risiko für das Nichterreichen des Ziels zwischen Netz und Versicherer aufgeteilt wird. Dass vier Fünftel der Ärztenetze bzw. die darin zusammengeschlossenen Ärzte teilweise seit vielen Jahren - eine Form der BMV anwenden, unterstreicht die Sinnhaftigkeit und Akzeptanz dieses Zusammenarbeitsprinzips zwischen Ärztenetzwerken und Versicherern.

\section{Was ist ein Budget?}

Ein Budget ist eine Kostenzielvorgabe für die Betreuung eines Versichertenkollektivs. Dabei sind die Ärzte beteiligt an Gewinn oder Verlust bei Erreichen/Nichterreichen der Zielvorgabe. Ein solches Budget wird immer zwischen dem Ärztenetz und den Versicherern ausgehandelt und deckt in der Regel alle Leistungen der Obligatorischen Krankenpflegeversicherung ab (ambulant und stationär). Budgetmitverantwortung bedeutet: Ein Ärztenetz übernimmt die Verantwortung, dass für eine bestimmte Gruppe von Patienten ein vereinbartes Kostenziel eingehalten wird. Dabei wird vor allem die (finanzielle) Risikobeteiligung, deren prozentuale Aufteilung zwischen Versicherer und Ärztenetz und - häufig - eine Risikolimite für das Ärztenetz vertraglich geregelt [1]

\section{Wie wird ein Budget berechnet?}

Das Budget wird immer auf das gesamte Netz und nicht auf den einzelnen Arzt berechnet. Zur Budget- berechnung haben sich in der Schweiz mehrere Methoden bewährt:

- ein Vergleich des Managed-Care-Versichertenkollektivs mit einem konventionell versicherten Kollektiv (Parallelkostenrechnung);

- ein Vergleich des Managed-Care-Kollektivs mit seinen Vorjahreskosten (Vorkostenrechnung);

- eine Kopfpauschale (Capitation): der Betrag, den ein einzelner Versicherter voraussichtlich im kommenden Jahr in Abhängigkeit von Alter, Geschlecht, Wohnort und Gesundheitszustand benötigt. Die Summe der Kopfpauschalen ergibt dann das Budget für das Ärztenetz [2].

\section{Wie werden Rechnungen für ärztliche Leistungen gestellt bzw. bezahlt?} Budgets von Ärztenetzen sind in den meisten Fällen eine Parallel- oder Schattenrechnung, in der alle von den Versicherern bezahlten Rechnungen verbucht werden. Die Rechnungsstellung durch die Ärzte, deren Bezahlung und die Rückvergütung durch die Versicherer erfolgen gleich wie im konventionellen System.

\section{Wohin geht ein Gewinn bzw. ein Verlust?}

Ein Gewinn bzw. Verlust errechnet sich aus dem vereinbarten Budget abzüglich der effektiv bezahlten Rechnungen. Liegt ein Gewinn vor, wird dieser zwischen Ärztenetz und Versicherer geteilt. Auch ein Verlust wird geteilt, wobei das Ärztenetz je nach Rechtsform maximal mit seinem Eigenkapital haftet.

\section{Literatur}

1 Götschi AS, Weber A. Ein Budget für Ärzte? Schweiz Ärztezeitung. 2004;85(47):2498-503.

2 Dreiding P, Fritschi J. Warum Begriffe aus der Ökonomie uns Ärztinnen und Ärzte nicht erschrecken müssen! Schweiz Ärztezeitung. 2006;87(29/30):131-9. 